\title{
Diagnóstico y manejo de invaginación intestinal en población pediátrica
}

\section{Diagnosis and Management of Intussusception in Pediatric Population}

Pág. 66,74

Recibido: 09-05-2020

Aceptado: 19-06-2020

Dra. Viviana Ruiz Loría ${ }^{1}$

Dra. Sabrina Acosta Egea ${ }^{2}$

Dra. Marisabel Echeverría Miranda ${ }^{3}$

Dr. Esteban Salas Salas ${ }^{4}$

1. Hospital Metropolitano, San José, Costa Rica

2,3 Médico General, Trabajador independiente, San José, Costa Rica.

4. Hospital Dr. Rafael Ángel Calderón Guardia, San José, Costa Rica.

\section{RESUMEN}

La invaginación intestinal es la causa más frecuente de obstrucción intestinal y la segunda de abdomen agudo en la población pediátrica. Un $90 \%$ de los casos es primario o idiopático. Suele tener una triada de síntomas que incluyen: dolor abdominal, vómitos y presencia de sangre en heces. Generalmente, involucra el íleon el cual se invagina dentro del ciego por medio de la válvula ileocecal. Puede conducir a isquemia y luego perforación si no es tratada de forma correcta en un tiempo adecuado. La reducción por enema es un procedimiento efectivo y seguro para el tratamiento de la invaginación intestinal. El manejo inicial siempre es con enemas, sin importar si es una invaginación inicial o si es una recurrencia, antes de considerar opciones quirúrgicas, y usualmente resuelve un $85-90 \%$ de los casos.

\section{PALABRAS CLAVE}

Invaginación, pediátrico, ileo, enemas

\section{ABSTRACT}

Intestinal intussusception is the most frequent cause of intestinal obstruction and the second cause of acute abdomen in the pediatric population. Ninety percent of cases are primary or idiopathic. It usually has a triad of symptoms including: abdominal pain, vomiting, and blood in the stool. Generally, it involves the ileum 
which is invaginated into the cecum through the ileocecal valve. It can lead to ischemia and then perforation if it is not treated correctly in a short amount of time. Rectal enema reduction is an effective and safe procedure for the treatment of intestinal invagination. Initial management is always with enemas, regardless of whether it is an initial invagination or a recurrence, before considering surgical options, and usually resolves $85-90 \%$ of cases.

\section{KEY WORDS}

Intussusception, pediatric, ileum, enemas

\section{INTRODUCCIÓN}

La invaginación intestinal es una de las causas más comunes de dolor abdominal agudo en la población pediátrica, al igual que la fuente número uno de obstrucción intestinal en niños e infantes $(1,2,3,4,5,6,7,8)$. Cuando un paciente pediátrico se presente a emergencias con dolor abdominal agudo siempre hay que descartar una invaginación intestinal dentro de las causas abdominales $(2,3,4)$. Esta sucede cuando un segmento proximal del intestino se adentra o invagina en un segmento intestinal distal adyacente $(1,7,9)$. Las invaginaciones ileocólicas representan un $80 \%$ y un $90 \%$ de invaginaciones intestinales se dan a raíz de causas idiopáticas $(6,7,8)$. Es una patología difícil de diagnosticar lo que puede llevar a un diagnóstico tardío y puede conllevar a complicaciones como isquemia, necrosis, perforación y hasta peritonitis $(1,8,10)$.

Esta revisión tiene como objetivo exponer evidencia actualizada sobre la epidemiología, diagnóstico y tratamiento de la invaginación intestinal para resaltar la importancia de un diagnóstico temprano y evitar posibles complicaciones letales.

\section{MÉTODOS}

La presente revisión bibliográfica se realizó mediante la recopilación y análisis de estudios y artículos basados en evidencia, seleccionados de las bases de datos de Elsevier, Pubmed y Google Scholar. Se utilizaron términos como "invaginación”, "intestinal”, "manejo” y "pediatría”. Se seleccionó literatura en inglés y español. Se utilizó como criterios de inclusión la validez de dichas fuentes bibliográficas por hospitales reconocidos a nivel mundial, artículos y estudios realizados por médicos especialistas en el área y que haya sido realizado en los últimos 5 años en el periodo entre el 2015-2020. Por lo tanto, se excluyeron todas las fuentes con antigüedad anterior al 2015.

\section{EPIDEMIOLOGÍA}

Esta patología puede suceder en niños de cualquier edad; sin embargo, tiene una predominancia por edades jóvenes, sobre todo antes de los 3 años de vida $(1,2,3,6)$. Suele tener una incidencia de 1 a 4 casos por cada 1000 nacidos vivos y tiene una proporción de 2:1 según la razón de hombres: mujeres $(1,5,6,7)$. Dentro del tipo más común de invaginación, se encuentran las invaginaciones ileocólicas; estas representan un $80 \%$ y se realizan cuando la porción del íleo terminal se adentra en el colon ascendente o ciego por medio de la válvula ileocecal $(1,6,9)$. Sin embargo, una invaginación intestinal puede llegar a ocurrir en cualquier parte del intestino (9). De hecho, al contrario de las invaginaciones ileocólicas, las ileoileales o de intestino delgado con intestino delgado no siempre requieren de tratamiento ya que pueden ser transitorias y resolver de forma esporádica (11). La mortalidad varía desde un $1 \%$ en países desarrollados a un $10 \%$ en países subdesarrollados, ya que estas situaciones 
influyen en el tiempo transcurrido a un centro especializado y en el manejo proporcionado $(6,14)$.

\section{CAUSAS}

Alrededor de un $90 \%$ de invaginaciones intestinales se dan a raíz de causas idiopáticas y es rara su asociación con patologías como divertículo de Meckel, púrpura de HenochSchonlein, apendicitis, pólipos intestinales o linfomas $(1,2,3,5,9,14)$. Se ha visto mediante estudios recientes que niños mayores de 3 años suelen asociar una lesión patológica o maligna causante de la invaginación en un 1.5 a $12 \%$ de los casos, por lo que suelen requerir de manejo quirúrgico $(2,5,7,9)$.

Hay ciertos casos anatómicos que contribuyen a la formación y eventual desarrollo de una invaginación intestinal, dentro de estas se incluyen: inserción anterior del íleo terminal con respecto al ciego, disminución de la rigidez del ciego secundario a presencia de tenias y la presencia de fibras musculares longitudinales del colon en la válvula ileocecal (9). Otras condiciones que favorecen la aparición de esta patología son las infecciones virales, alergias, enfermedad de Crohn y la hipertrofia de la placa de Peyer $(7,9)$.

\section{DIAGNÓSTICO}

La intususcepción puede ser difícil de diagnosticar porque la tríada clásica de síntomas (cólico abdominal, masa abdominal y heces de grosella roja) es evidente en el momento de la presentación en sólo alrededor del $20 \%$ de los casos $(7,8,13,14)$. Aunque es una de las causas más comunes de abdomen agudo en los lactantes y los niños pequeños, a menudo plantea un desafío diagnóstico para el médico en el momento de la primera presentación porque los síntomas suelen ser inespecíficos y pueden ser bastante variables $(1,7,13)$. Esto puede llevar a retrasos en la realización del diagnóstico correcto (13). Los síntomas de la invaginación intestinal se superponen a otros múltiples procesos de la enfermedad y la incapacidad de los niños pequeños para comunicar sus síntomas agrava el enigma clínico (1). Afortunadamente, el diagnóstico por imágenes de la invaginación intestinal ha sido bien descrito y puede hacerse con un alto grado de precisión (1).

Los niños con intususcepción suelen estar previamente asintomáticos antes de desarrollar un inicio repentino de dolor abdominal tipo cólico y emesis que progresa a emesis biliar (14). Las características más comunes que se presentan son dolorabdominal con cólicos, vómitos, palidez, letargo, diarrea, fiebre, disentería y apatía $(3,8,13)$. Estos síntomas en los recién nacidos prematuros cuando se constata una distensión abdominal son muy sugestivos de enterocolitis necrotizante, la emergencia gastrointestinal adquirida más común en la unidad de cuidados intensivos neonatales, lo cual puede conllevar a un retraso en el tratamiento de los pacientes (10).

El hallazgo abdominal clave en la intususcepción es la palpación de una masa en forma de "salchicha" en el abdomen, pero una vez que los síntomas han progresado más allá de unas 24 horas, esto se hace menos evidente porque la distensión del intestino gaseoso superpuesto por la obstrucción del intestino delgado lo obstruye (13). La diarrea tiende a ser breve y de pequeño volumen a medida que el colon se vacía distalmente a la obstrucción, a diferencia de la gastroenteritis (13). Además, sugieren que la intususcepción es probablemente bastante

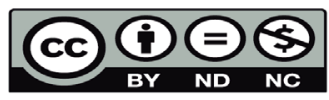


estrecha, lo que puede reflejarse en la menor tasa de éxito del intento de reducción del enema (13).

En enfermedades avanzadas, como cuando la intususcepción ha estado presente durante 3-4 días, las características son más de septicemia y deshidratación, pero la pista del diagnóstico subyacente es la adición de dolor abdominal y distensión y/o sensibilidad abdominal (13). El paso de heces rojas gelatinosas (debido a la necrosis de la mucosa) con una distensión abdominal progresiva, aunque poco común, son indicativos de una enfermedad avanzada y hace el diagnóstico bastante claro $(13,14)$. El suministro de sangre está comprometido y la isquemia intestinal y la perforación resultan de la gangrena de la pared del intestino (14). El retraso en la presentación después de 24 horas de la aparición de los síntomas predispone a complicaciones intestinales y a una mayor necesidad de intervención quirúrgica primaria, y se ha demostrado que está asociado a tasas de mortalidad más elevadas (14). Puede conducir a isquemia y perforación si no es tratada de forma correcta en un tiempo adecuado (6).

En losúltimos años, los algoritmos de manejo dela intususcepción han cambiado significativamente ya que la radiografía abdominal simple ha sido reemplazada por la ultrasonografía (13). Esto ya no es así porque la ecografía es una modalidad de diagnóstico por imágenes mucho más fiable y precisa para diagnosticar la intususcepción que la radiología simple, encontrar una obstrucción intestinal en una radiografía no es una contraindicación a la reducción del enema per se, y el neumoperitoneo en la intususcepción previo a un enema prácticamente nunca sucede en el mundo occidental (13). Además, la placa abdominal plana no suele ser útil para el diagnóstico de la intususcepción neonatal (10).
En la mayoría de los hospitales, la ecografía se considera ahora el estándar de oro para confirmar el diagnóstico de intususcepción $(13,14)$. Tiene una alta sensibilidad y especificidad para la detección de la intususcepción $(97,9 \%$ y $97,8 \%$, respectivamente), así como un alto valor predictivo negativo $(99,7 \%)(1,14)$. Este método diagnóstico es barato y fácil de realizar y no implica ninguna radiación, lo cual lo hace ideal para pacientes pediátricos $(1,13)$. El ultrasonido abdominal revelaría una imagen característica conocida como "signo del tiro al blanco" (4).

El ultrasonido Doppler contribuye al diagnóstico de la intususcepción de varias maneras (1). Se ha demostrado que la ausencia de flujo sanguíneo en la intususcepción se correlaciona con la isquemia y la necrosis intestinal en la cirugía y es un factor de predicción de una reducción de enema infructuosa (1). Es un hallazgo importante junto con el estado clínico general del niño para determinar el tratamiento adecuado (1). Además de las características Doppler, se pueden utilizar otros múltiples hallazgos para predecir la probabilidad de éxito en la posterior reducción del enema, los cuales pueden reflejar una intususcepción encarcelada: líquido intraperitoneal libre, neumatosis, líquido atrapado dentro de la intususcepción y obstrucción del intestino delgado (1).

EI POCUS (ultrasonido en el punto de atención) realizado utilizando los criterios establecidos para estándares más amplios por los médicos de emergencias pediátricas puede ser útil para detectar la intususcepción en una etapa temprana, que puede presentar síntomas clínicos inespecíficos (8). POCUS puede ser realizado por médicos de emergencias pediátricas para detectar la intususcepción (8).

Históricamente, el enema con bario, aire o medio 
de contraste soluble en agua se usaba para diagnosticar una sospecha de intususcepción $(1,2)$. La introducción de un medio de contraste en el colon muestra la intususcepción como un defecto de relleno intraluminal en forma de media luna o redonda, y la ubicación y el aspecto de las imágenes del borde anterior de la intususcepción pueden predecir la probabilidad de una reducción exitosa (1). Las deficiencias del enema de diagnóstico incluyen la detección limitada de otras causas de dolor abdominal agudo, la baja sensibilidad para las intususcepciones de intestino delgado, la técnica invasiva y la exposición a la radiación ionizante (1). Una ventaja del diagnóstico de la intususcepción por enema es la posibilidad de conversión inmediata a un procedimiento terapéutico (1). El enema de contraste rectal no debe utilizarse en un paciente neonatal debido a la vulnerabilidad de los intestinos a la perforación (10).

\section{DIAGNÓSTICOS DIFERENCIALES}

Debido a que la presentación clínica de la intususcepción puede ser inespecífica, el radiólogo debe considerar un amplio diagnóstico diferencial para el dolor abdominal agudo del niño (1). Cuando un paciente pediátrico se presenta a emergencias con dolor abdominal agudo siempre hay que descartar una invaginación intestinal dentro de las causas abdominales (4).

En la África Sub-sahariana, la intususcepción temprana tiene un amplio diagnóstico diferencial que incluye paludismo, gastroenteritis $y$ disentería, todos ellos más frecuentes que la intususcepción, lo que complica el diagnóstico y el tratamiento temprano en estas áreas (14).

Otros diagnósticos diferenciales son: gastroenteritis perola diarrea en la intususcepción es de poco volumen y corta duración, a diferencia de la que se ve en la gastroenteritis (13). Septicemia donde la pista de una causa intraabdominal es distensión y sensibilidad abdominal (13). Hernia inguinal estrangulada la cual es evidente si hay un bulto inguinal irreducible (13). La enfermedad de Hirschsprung donde hay una edad comparativamente tardía de aparición de los síntomas y debe haber un antecedente de estreñimiento severo u obstrucción (13). Infección del tracto urinario donde se vería la orina clara (13). Apendicitis aguda, ésta puede ocurrir en los primeros 2 años de vida pero es raro (13). Las características abdominales pueden ser similares, con un dolor abdominal generalizado y peritonismo (13). El apéndice perforado y la colección contenida adyacente pueden tener la clásica apariencia de capas concéntricas alternas de tejidos hiper e hipoecoicos con un centro ecogénico, con contenido entérico o un apéndice que imita la grasa mesentérica dentro de una intususcepción (1). La ultrasonografía normalmente puede distinguir entre las dos condiciones (13). El divertículo de Meckel es otro diagnóstico diferencial (1).

Un hallazgo muy específico de la intususcepción es la presencia de nódulos linfáticos mesentéricos dentro del lumen del intususcipiente (1). La identificación de los ganglios linfáticos dentro de la lesión en cuestión sugiere fuertemente la invaginación intestinal como diagnóstico, y podría ayudar a distinguir entre la verdadera invaginación intestinal y sus diagnósticos diferenciales (1). Esto es especialmente cierto en la distinción entre las intususcepciones ileocólicas y del intestino delgado, que no contienen ganglios linfáticos ni grasa mesentérica y suelen tener menos de $2 \mathrm{~cm}$ de diámetro (1). Además, las intususcepciones idiopáticas del intestino delgado son a menudo transitorias $(1,11)$. Si se detecta una durante un examen, se suele demostrar la resolución de la

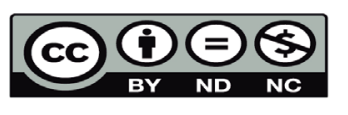


intususcepción del intestino delgado, mientras que una intususcepción ileocólica sintomática suele persistir (1).

\section{TRATAMIENTO}

El tratamiento de la invaginación intestinal depende del tipo de invaginación y la estabilidad del paciente $(15,16)$. Actualmente el tratamiento se divide en un manejo quirúrgico y no quirúrgico $(15,16)$.

La invaginación ileocólica es la más común en la edad pediátrica y se tiende a manejar con una reducción guiada por ultrasonido (15). Las invaginaciones del intestino delgado, que no son comunes en la edad pediátrica, normalmente se reducen espontáneamente (15). Sin embargo, si éstas persisten, se asocian a necrosis intestinal y se necesitará una intervención quirúrgica (15). Es necesario mantener al paciente en observación posterior al tratamiento inicial, ya que hay una alta posibilidad de recurrencia en las primeras 24 horas (15). Un manejo quirúrgico se requiere siempre que haya datos de necrosis o perforación intestinal, peritonitis, shock, o cuando el manejo no quirúrgico fracase $(15,16)$. La invaginación intestinal es una de las emergencias más comunes en niños menores a 3 años, por lo tanto es imprescindible conocer su adecuado manejo (17).

\section{MANEJO NO QUIRÚRGICO}

Un $85-90 \%$ de los casos de invaginación intestinal se pueden solucionar con un manejo no quirúrgico (16). Este consiste en colocar un medio de contraste en el recto (sea aire, solución salina o bario), para reducir la invaginación al aumentar la presión intraluminal (17). Se utiliza fluoroscopia o ultrasonografía para guiar el contraste y monitorizar la reducción (17). Sin embargo, se apoya el uso del ultrasonido ya que este elimina la exposición a radiaciones ionizantes y permite un monitoreo continuo del movimiento retrógrado de la invaginación (15).

Se aprecia un flujo libre del medio de contraste hacia el íleo cuando se llega a realizar adecuadamente la reducción (15).

El uso de tratamiento adyuvante farmacológico es controversial, ya que no queda clara su eficacia, sin embargo se cree que facilita el manejo no quirúrgico del paciente (17). Actualmente los medicamentos adyuvantes que se están usando son el glucagón y los antibióticos (17). El glucagón reduce el tono muscular del colon y provee analgesia, sin embargo, un estudio reciente sugiere que este medicamento no aumenta la tasa de reducción en el manejo no quirúrgico de pacientes con invaginación intestinal (17). El uso de antibióticos también es controversial, ya que se ha reportado un riesgo aumentando de invaginación intestinal posterior al uso de estos medicamentos (17). Hay bajo riesgo de bacteremia al realizar una reducción con aire guiada por fluoroscopia y por lo tanto no se recomienda el uso de antibióticos en estos casos (17). Actualmente el manejo no quirúrgico conservador es el estándar de tratamiento para la invaginación intestinal en pacientes pediátrico (15). Factores como sangrado por el recto, síntomas de larga duración o la edad menor del paciente reduce el porcentaje de éxito de la reducción por enema (15). Sin embargo, siempre se recomienda un intento inicial de manejo no quirúrgico (15).

\section{MANEJO QUIRÚRGICO}

Actualmente existen cuatro indicaciones absolutas para tratar quirúrgicamente la invaginación intestinal (13). Estas indicaciones consisten en; peritonitis o evidencia clínica de 
necrosis intestinal, la ineficacia de la reducción no quirúrgica aun tras realizar repetidos enemas, una fuerte sospecha de que la invaginación es causada por una lesión patológica como el síndrome de Peutz Jegher, y para un abdomen agudo en que el diagnóstico pre quirúrgico no es claro y se debe realizar una laparoscopía o laparotomía para revelar la invaginación (13). La causa más común para el manejo quirúrgico es por una falta de resolución por parte del manejo no quirúrgico y en estos casos es común que se necesite realizar una resección intestinal donde se encuentra la invaginación (13).

La cirugía consiste en una laparotomía abierta con una incisión supraumbilical transversal derecha $(13,17)$. Se realiza una reducción manual de la invaginación y solo en casos de necrosis, perforación o inhabilidad de realizar la reducción se resecaría la porción intestinal afectada $(13,17)$. Actualmente se ha demostrado que la laparoscopía puede ser una opción quirúrgica más segura, con una hospitalización menor e igual de efectiva que la laparotomía abierta $(17,18)$. Sin embargo, generalmente los casos en que se decide por un manejo quirúrgico son aquellos de larga duración, donde el intestino se encuentra dilatado, o aquellos que presentan necrosis y se va a necesitar realizar una resección de la porción afectada (13). Por lo tanto, realizar una laparoscopía en dichos casos puede ser complejo para el cirujano, ya que el intestino dilatado compromete la adecuada visualización del espacio quirúrgico y se aumenta el riesgo de necesitar una conversión a laparotomía abierta para efectuar una adecuada resección intestinal $(13,18)$. Sin embargo, a pesar de estas limitaciones se recomienda intentar en primera instancia una reducción laparoscópica (18).

\section{PRONÓSTICO}

Actualmente el porcentaje de recurrencia para la invaginacion intestinal es de aproximadamente $10 \%$, con $0 \%-0.6 \%$ en las primeras 24 horas tras realizar un manejo conservador, comparado con un $0,4 \%$ tras una reducción quirúrgica (15). Los factores de riesgo para una recurrencia no son claros y el manejo de estos episodios es controversial (15). Esta enfermedad tiene un buen pronóstico, siempre y cuando se realice un diagnóstico oportuno y un tratamiento adecuado (15).

\section{CONCLUSIÓN}

La invaginación intestinal en niños es una patología frecuente y que todo médico que valore pacientes pediátricos debe considerar como diagnóstico diferencial en el momento de una valoración inicial por dolor abdominal. Es importante tener en cuenta el predominio en los pacientes menores de 3 años, los cuales presentan un desafío clínico con síntomas inespecíficos. Afortunadamente se cuenta con el ultrasonido como método diagnóstico el cual es muy específico y sensible. La mayoría de los casos se manejan de manera no invasiva evitando realizar un procedimiento quirúrgico. Se debe tener siempre en cuenta el diagnóstico de causas de dolor abdominal con posibles consecuencias catastróficas, como la invaginación intestinal, ya que si no se diagnostica a tiempo, conlleva a isquemia con posible necrosis y perforación. Cabe resaltar que esta enfermedad tiene un buen pronóstico pero que puede tener desenlaces que resultan en una alta morbi-mortalidad.

\section{CONFLICTOS DE INTERÉS}

Los autores declaran que no existen conflictos de intereses ni beneficios económicos al realizar este artículo.

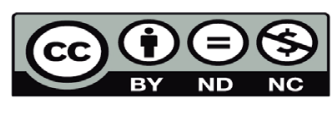




\section{BIBLIOGRAFÍA}

1. Edwards EA, Pigg N, Courtier J, Zapala MA, MacKenzie JD, Phelps AS. Intussusception: past, present and future. Pediatric radiology. 2017 Aug 1;47(9):1101-8. DOI: 10.1007/s00247-017$3878-x$

2. Savoie KB, Thomas F, Nouer SS, Langham Jr MR, Huang EY. Age at presentation and management of pediatric intussusception: a Pediatric Health Information System database study. Surgery. 2017 Apr 1;161(4):995-1003. DOI: 10.1016/j.surg.2016.09.030

3. Mehendale S, Kumar CG, Venkatasubramanian S, Prasanna T. Intussusception in children aged less than five years. The Indian Journal of Pediatrics. 2016 Oct 1;83(10):1087-92. DOI: 10.1007/s12098-016-2152-9

4. Sharon MJ, End B, Findley S, Kraft C, Minardi JJ. Definitive Diagnosis before Leaving the Room: POCUS for Pediatric Intussusception. Journal of Emergency Medicine. 2019 Aug 1;57(2):247-8. DOI: 10.1016/j.jemermed.2019.03.040

5. Wang A, Prieto JM, Ward E, Bickler S, Henry M, Kling K, Thangarajah H, Ignacio Jr R. Operative treatment for intussusception: Should an incidental appendectomy be performed?. Journal of pediatric surgery. 2019 Mar 1;54(3):495-9. DOI: 10.1016/j.jpedsurg.2018.10.099

6. Trang NV, Burnett E, Ly LH, Anh NP, Hung PH, Linh HM, Trang NC, Canh TM, Minh VT, Tate JE, Yen C. Recurrent intussusception among infants less than 2 years of age in Vietnam. Vaccine. 2018 Dec 14;36(51):7901-5. DOI: 10.1016/j.vaccine.2018.02.056

7. Shiyi EY, Ganapathy S. Intussusception in children presenting to the emergency department: an Asian perspective. Pediatric emergency care. 2017 Jun 1;33(6):409-13.

8. Lee JY, Kim JH, Choi SJ, Lee JS, Ryu JM. Point-of-care ultrasound may be useful for detecting pediatric intussusception at an early stage. BMC pediatrics. 2020 Dec;20(1):1-6.

DOI: 10.1186/s12887-020-02060-6

9. Tafner E, Tafner P, Mittledorf C, Pinhata J, Silva AL, Pilli S, da Silva JG, Hassegawa RT, Maruta L, Christiano C, Andrada L. Potential of colonoscopy as a treatment for intussusception in children. Endoscopy international open. 2017 Nov;5(11):E1116-8.DOI: 10.1055/s-0043-117950

10. Aydin E. Intussusception in a preterm newborn. Pediatrics \& Neonatology. 2018 Jun 1;59(3):3124. DOI: 10.1016/j.pedneo.2017.08.009

11. Melvin JE, Zuckerbraun NS, Nworgu CR, Mollen KP, Furtado AD, Manole MD. Management and Outcome of Pediatric Patients With Transient Small Bowel-Small Bowel Intussusception. Pediatric emergency care. 2018 May. 
12. Okumus $M$, Emektar A. Pediatric intussusception and early discharge after pneumatic reduction. Acta Chirurgica Belgica. 2019 May 4;119(3):162-5. DOI: 10.1080/00015458.2018.1487190

13. Beasley SW. The 'ins' and 'outs' of intussusception: Where best practice reduces the need for surgery. Journal of paediatrics and child health. $2017 \mathrm{Nov} ; 53(11): 1118-22$.

DOI: $10.1111 /$ jpc. 13738

14. Akello VV, Cheung M, Kurigamba G, Semakula D, Healy JM, Grabski D, Kakembo N, Ozgediz D, Sekabira J. Pediatric intussusception in Uganda: differences in management and outcomes with high-income countries. Journal of pediatric surgery. $2019 \mathrm{Jul} 15$.

DOI: 10.1016/j.jpedsurg.2019.07.003

15. Caruso AM, Pane A, Scanu A, Muscas A, Garau R, Caddeo F, Mascia L. Intussusception in children: not only surgical treatment. Journal of Pediatric and Neonatal Individualized Medicine (JPNIM). 2017 Feb 25;6(1):e060135. DOI: 10.7363/060135

16. Marsicovetere P, Ivatury SJ, White B, Holubar SD. Intestinal intussusception: etiology, diagnosis, and treatment. Clinics in colon and rectal surgery. 2017 Feb;30(01):030-9.

DOI: $10.1055 / \mathrm{s}-0036-1593429$

17. Gluckman S, Karpelowsky J, Webster AC, McGee RG. Management for intussusception in children.Cochrane Database of Systematic Reviews. 2017(6).

DOI: 10.1002/14651858.CD006476.pub3.

18. Benedict LA, Ha D, Sujka J, Sobrino JA, Oyetunji TA, St. Peter SD, Fraser JD. The Laparoscopic Versus Open Approach for Reduction of Intussusception in Infants and Children: An Updated Institutional Experience. Journal of Laparoendoscopic \& Advanced Surgical Techniques. 2018 Nov 1;28(11):1412-5.

DOI: 10.1089/lap.2018.0268 